Proceedings of the 2011 Winter Simulation Conference

S. Jain, R.R. Creasey, J. Himmelspach, K.P. White, and M. Fu, eds.

\title{
SIMULATION OF MITIGATION STRATEGIES FOR A PANDEMIC INFLUENZA
}

\author{
Arsalan Paleshi \\ Gerald W. Evans \\ Sunderesh S. Heragu \\ Kamran S. Moghaddam \\ University of Louisville \\ 220 Eastern Parkway \\ Louisville, KY 40292, USA
}

\begin{abstract}
Millions of people have been infected and died as results of influenza pandemics in human history. In order to prepare for these disasters, it is important to know how the disease spreads. Further, intervention strategies should be implemented during the pandemics to mitigate their ill effects. Knowledge of how these interventions will affect the pandemic course is paramount for decision makers. This paper develops an agent-based simulation model of a pandemic within a generic US metropolitan area, along with the effects associated with mitigation strategies involving home confinement and school closure. Also, a comparison of the two strategies and their variants is presented.
\end{abstract}

\section{INTRODUCTION}

Three kinds of influenza virus have been found so far, influenza A, B, and C. Influenza A is the most dangerous one among them. It has caused three major pandemic influenzas during the $20^{\text {th }}$ century. The first one, Spanish flu, occurred in 1918-1920 and killed 40 million people worldwide (Gatherer 2009). The second one called Asian flu occurred in 1957-1958 and caused 68,900 mortalities in the United States. Finally the third one, Hong Kong flu, occurred in 1968-1969 and 33,800 individuals died as a result of this disease in the United States. The economic costs of the Hong Kong flu was approximately 3.9 billion dollars in the US (Hilleman 2002). The amount of damage caused by the influenza virus made it the most dangerous pandemic disease threat to human kind (Gatherer 2009). The world witnessed another pandemic in 2009 which was not as severe as the three mentioned above, but the threat of this disease still exists.

Large scale fatalities and economic losses of influenza pandemics highlight the importance of emergency preparedness for handling the consequences of these disasters. It is important for the authorities to employ the best intervention strategies such as school closure to mitigate the ill effects of a pandemic and also effectively plan for resource allocation (Ferguson et al. 2006).

One way to evaluate intervention strategies is to simulate the course of the pandemic and analyze the effects of the strategies. Researchers have addressed the problem of simulating the behavior of influenza pandemic by two main approaches, namely differential equation simulation models and agent-based simulation models. In the first approach, a compartmental Susceptible, Infective, Recovered (SIR) model or one of its variants are developed which divides the population into three groups - people susceptible to the virus, infectious people, and individuals who recover from the disease. Following this, differential equation based relations between the number of people in these three groups through time is developed and used to calculate the number of infections. Towers and Feng (2009) use a deterministic SIR model to predict the course of pandemic H1N1 2009 flu during fall of 2009 using the data on the number of infec- 


\section{Paleshi, Evans, Heragu, and Moghaddam}

tions in summer 2009. They also evaluate the effectiveness of a vaccination program planned for autumn of that year. Extensions of the SIR model have been used in other studies. For example, Dushoff et al. (2004) develop an SIR-Susceptible (SIRS) model which lets the recovered person become susceptible again. Due to the possibility of the virus mutation during the pandemic an SIRS system is capable of modeling these situations. In another study, Araz et al. (2010) use differential equations to simulate the spread of the avian influenza (H5N1) in the counties of Arizona. The model predicts the number of infected and dead people in different age groups over time during a pandemic influenza. Differential equation simulation models are insightful and relatively fast tools for simulating pandemics, but they have some major pitfalls. In order to evaluate the effectiveness of intervention strategies, it is important to assess their effects on different age groups, communities or even some special individuals. Also, the transmission rate of the disease between individuals and progress of the disease within the body have probabilistic natures that should be considered. Differential equation simulation models are not the best tools to address these requirements.

Another approach to simulate the pandemic influenza spread which does not have the drawbacks of mathematical based models is agent-based modeling simulation (ABMS). Each individual is considered as an agent in this modeling approach and interactions of the people are modeled in different locations such as households, schools, work places. Having each individual as an independent agent in the simulation gives a high level of flexibility to the model. Elveback et al. (1976) is one of the first studies that uses ABMS to predict the course of a pandemic and evaluates the effectiveness of school closure and vaccination mitigation strategies. Longini et al. (2004) simulate the spread of influenza A within a US community with 2000 people. The population is divided into four neighborhoods with two elementary schools, one middle school, and one high school. The work by Longini et al. (2004) has many similarities to Elveback et al. (1976), but it is a step forward and considers more details. In another study, Shi et al. (2010) develop an agent-based simulation model to evaluate the effects of seasonal changes of virus characteristics on the pandemic course. Aleman, Wibisono, and Schwartz (2009) consider the Greater Toronto Area in Canada as the target population and develop an agent-based model. This model evaluates the effects of a home confinement strategy on the number of infected and dead people during a pandemic.

Infectious people might show symptoms of disease or be asymptomatic (Wu et al. 2006). In addition, the relative infectious rate is different for symptomatic people than asymptomatic ones. A 63 percent probability of being symptomatic (PBS) is considered in the literature (Elveback et al. 1976). A change in the value of this parameter can highly effect the number of infected people during a pandemic and change the effectiveness levels of intervention strategies.

In this paper, an agent-based simulation model is developed to predict the course of a pandemic over time. Home confinement and school closing intervention strategies are presented and their effects on the number of infections are assessed. They are also compared with each other and a sensitivity analysis on the probability of being symptomatic and its impact on the number of infections is conducted.

This paper is organized as follows: Section 2 presents the developed agent-based simulation model; Section 3 explains the details of proposed home confinement and school closing strategies. Section 4 presents the results of the simulation models with three scenarios, namely baseline scenario without interventions, home confinement and school closure. Section 5 depicts the sensitivity analysis of the simulation model on the probability of being symptomatic and finally Section 6 concludes the paper and makes suggestions for future research.

\section{AN AGENT-BASED SIMULATION MODEL OF PANDEMIC SPREAD}

An influenza outbreak is simulated in a US metropolitan area. Data on the influenza A (H2N2) pandemic in 1957-58 are used for the disease characteristics' related aspects of the model according to Haber et al. (2007). The population structure of the model is based on the data from 2000 US Census. The model consists of three main sub-routines - the structure of the population which includes the mixing and age groups, disease characteristics and progress of the disease within the body, and transmission of the dis- 
ease between people. These three aspects of the simulation model are explained in more detail in this section.

\subsection{Population Structure}

People belong to one of four age groups; preschool children (less than or equal to 4 years old), school children (5 to 18 years old), adults (19 to 64 years old), and seniors (65 years old or older). There are five categories of mixing groups labeled households, daycare centers, schools, work places, and community. People interact with others in these groups. Disease is transmitted from an infectious person to a susceptible one during these contacts in the mixing groups. Each person belongs to a household and preschool individuals, students, and adults go to daycare centers, schools, work places, respectively. All the people are involved in the daily activities in the community (i.e., visiting stores, theaters and churches) as a whole. Table 1 illustrates the mixing and age groups considered in the model (Haber et al. 2007).

Table 1: Mixing and age group matrix

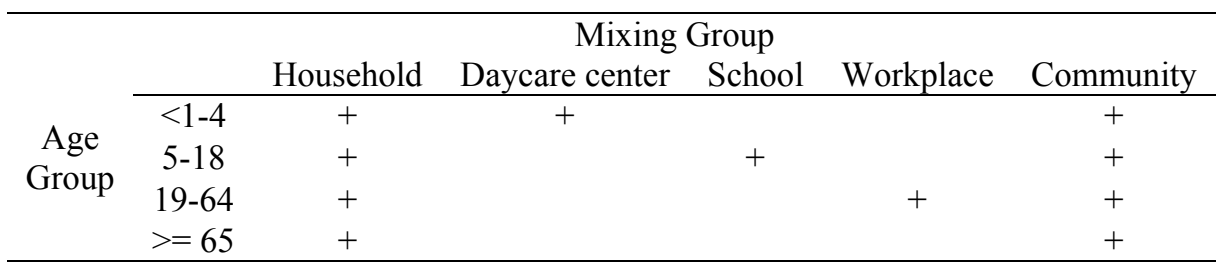

\subsection{Virus Characteristics and Disease Progress within the Body}

A susceptible person becomes infected as a result of having contact with an infectious person. After that, the disease progresses as illustrated in Figure 1. At the beginning of the process a patient passes through an incubation period which lasts 1.9 days on average. Then, he or she becomes infectious which lasts 4.1 days on average. An individual might be symptomatic or asymptomatic. A symptomatic patient might become hospitalized and even die. After the infectious period, the patient becomes immune to the disease if he or she does not die. For more information, see Longini et al. (2004).

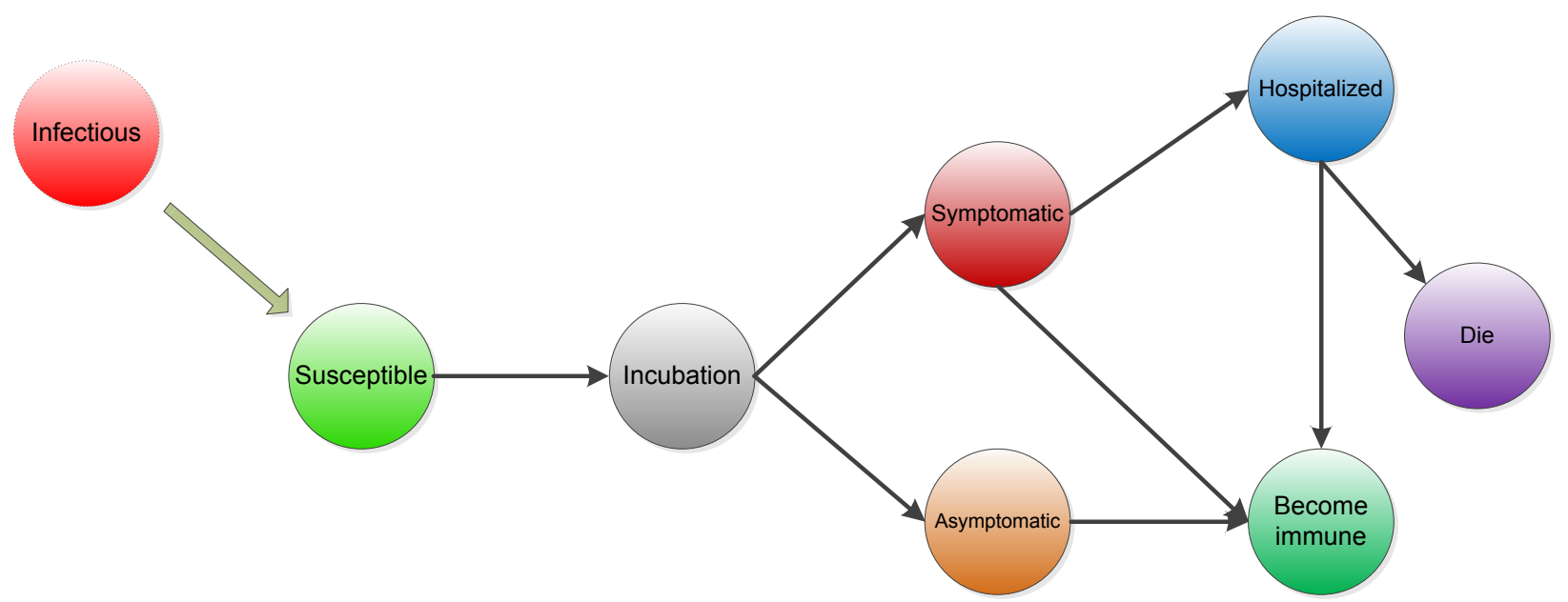

Figure 1: Progression of the disease in a susceptible person

\subsection{Disease Transmission Process}

Transmission of the disease to a susceptible individual depends on some parameters such as number and duration of contacts that a person has with infectious persons during daily activities and age specific 


\section{Paleshi, Evans, Heragu, and Moghaddam}

transmission rates of the disease from infectious individuals to him or her. Haber et al. (2007) present a formulation for deriving a probability of getting the disease on a day.

$$
P(A)=1-\Pi_{k} \Pi_{j} \Pi_{B \epsilon T_{A j k w}} \exp \left(-\lambda_{s j} d_{s_{A} j k w} M_{B}\right)
$$

In equation (1), $A$ is a susceptible individual in age group $s_{A}$ and $P(A)$ is the probability of individual $A$ becoming infected on a day. $T_{A j k w}$ denotes the individuals who have contacts with $A$ on a day (which might be a week day where $w=0$ or a weekend day where $w=1$ ) and are in mixing group $k$ and age group $j$. The rate of transmission per minute of contact from an infectious person in age group $j$ to individual $A$ is $\lambda_{s j}$ and duration of contact between person $A$ and a person in age group $j$ in mixing group $k$

on a day of type $w$ is $d_{s_{A} j k w}$ minutes. If individual $B$ is infectious, then $M_{B}$ is equal to one. Otherwise it is equal to zero. As shown in equation (1), the transmission rate of the disease changes when there is a change in the age group of infectious or susceptible persons. Also, contact durations depend on the mixing and age groups of the infectious and susceptible individuals and whether the contact happens on a week day or a weekend.

A numerical example of this formula is explained for a sample adult. There are two school students, one pre-school child, and one adult in his or her household. One of the school students is infectious and asymptomatic and the other family members are not infectious. There are nine co-workers in his or her workplace group from which one is infectious and symptomatic, one is infectious and asymptomatic, and the remaining co-workers are susceptible. The probability of this individual being infected during this day is calculated as follows.

There is one student in his household, who is infectious. The transmission rate of the disease from the school student to the adult is 0.00033 and the contact duration is 120 minutes. Also, an asymptomatic person is $50 \%$ less infectious than a symptomatic one. So, the probability of not being infected in household is $\alpha=\exp (-0.00033 * 120 * 0.5)$. The transmission rate of the disease from an adult to another one is 0.00032 and the contact duration in the work place is 120 minutes. So, the probability of not being infected at workplace is $\beta=\exp (-0.00032 * 120 * 0.5) * \exp (-0.00032 * 120)$.

Among the people in the community with whom this person has contacts, only one senior is infectious and asymptomatic and the remaining are not infectious. The transmission rate of the disease from a senior to an adult is 0.00029 and the contact duration in community between these two is 60 minutes. So, the probability of not being infected in the community is $\gamma=\exp (-0.00029 * 60 * 0.5)$. Finally, the probability of being infected for this adult during this weekday is $P_{\text {infection }}=1-\alpha * \beta * \gamma=1-$ $0.917502=0.082498$.

\subsection{Simulation Process}

A simulation program is developed in the JAVA programming environment and a high level flowchart of the simulation model is shown in Figure 2. The simulation begins with the generation of the individuals (i.e., agents). Agents are assigned to age groups and their disease characteristics are randomly determined. In addition, people are allocated to their mixing groups with attention to their age groups. The simulation is run for 35 weeks (245 days). In each day, people make contacts with the other individuals and at the end of each day the number of infected people is calculated. Then the simulation parameters are updated with the information from the current day and the model is run for the next day. At the end of the simulation run, the number of infected people is reported.

\section{INTERVENTION STRATEGIES}

Home confinement and school closure are intervention strategies examined in this paper with the aim of mitigation of the number of infected individuals during a pandemic outbreak. 


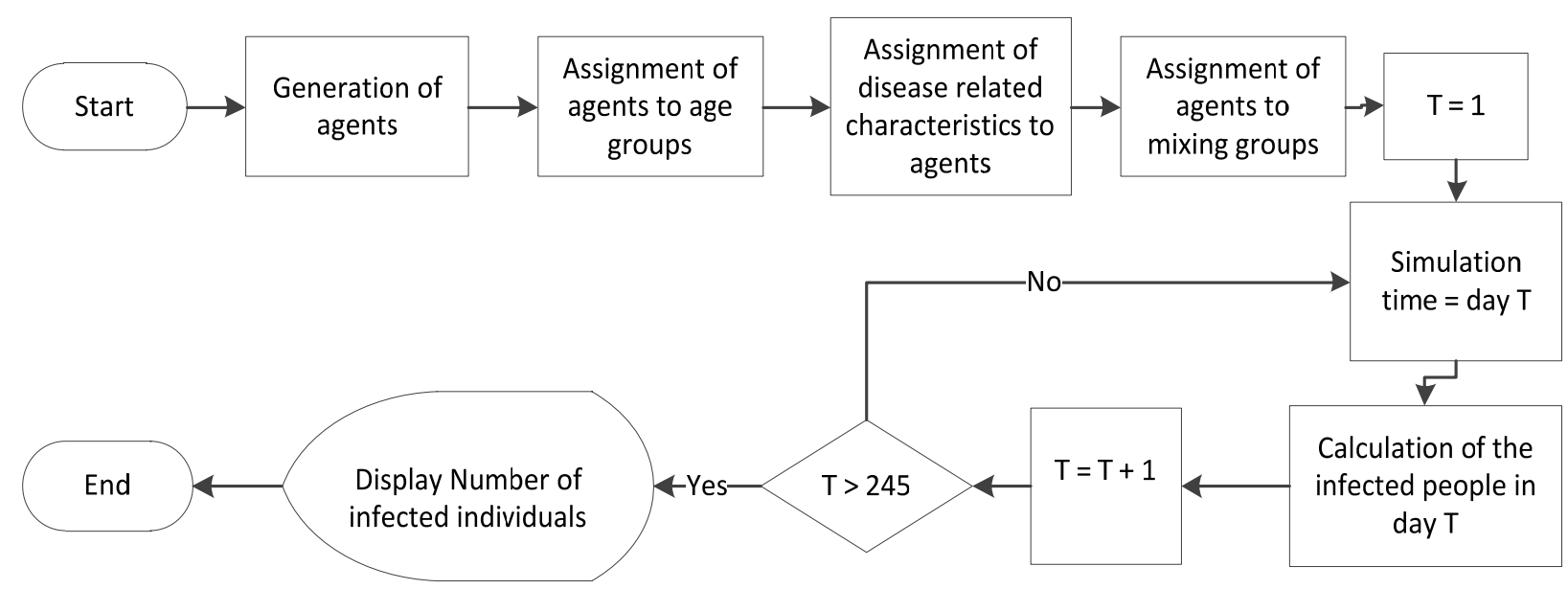

Figure 2: Structure and logic of the agent-based simulation model

\subsection{Home Confinement}

According to this strategy, a symptomatic infectious person stays home during the infectious period and only make contacts with his or her household members. Home confinement starts one day after a symptom appears and finishes one day after the illness ends. Duration of contacts between the confined person and other household members who continue to go to work or school do not change. Duration of contacts with susceptible household members who stay home is considered to be equal to the weekend values of contacts. It is reasonable to assume that only a fraction of the people comply with the confinement rules. The simulation is run for three compliance rates - 30\%, 40\%, and 50\% (Haber et al. 2007).

\subsection{School Closing}

Based on the school closing strategy, a school is closed for a certain period of time (i.e., one, two, or three weeks) when $5 \%$ of the students in that school show symptoms of disease. During school closure, household and community contact durations of the students whose schools are closed are considered to be equal to their weekend values. However, household contact durations between these students and their household members who continue to go to work or school do not change.

\section{COMPUTATIONAL RESULTS}

There are 50 replications for each of the scenarios. Figures 3, 4, and 5 illustrate the number of weekly infections during the pandemic course for baseline (without intervention), home confinement, and school closure strategies, respectively.

The attack rate (AR) of the disease is the percentage of infected people during the pandemic. In order to calculate the effectiveness of each strategy, formula (2) is used. Table 2 shows the effectiveness and attack rate of the pandemic for each of the scenarios.

$$
\text { Effectiveness }=\frac{[(\text { Baseline attack rate })-(\text { attack rate with intervention })]}{(\text { Baseline attack rate })} * 100 \%
$$

As shown in Table 2, all the intervention strategies have positive effects on the AR of the pandemic. A comparison of the intervention strategies' effectiveness indicates that home confinement with $50 \%$ compliance rate has the biggest effect on the attack rate of the pandemic and decreases it by $39.6 \%$, while the school closure for one week has the lowest effect on AR and decreases its value only by $9.8 \%$. Further, comparison of Figures 3, 4, and 5 indicate that the maximum number of people infected in a week decreases as a result of applying the strategies. The maximum infection in a week for baseline scenario is 
$5.2 \%$, while it is $3.6 \%$ and $4.9 \%$ for home confinement with $30 \%$ compliance rate, and school closure for one week, respectively.

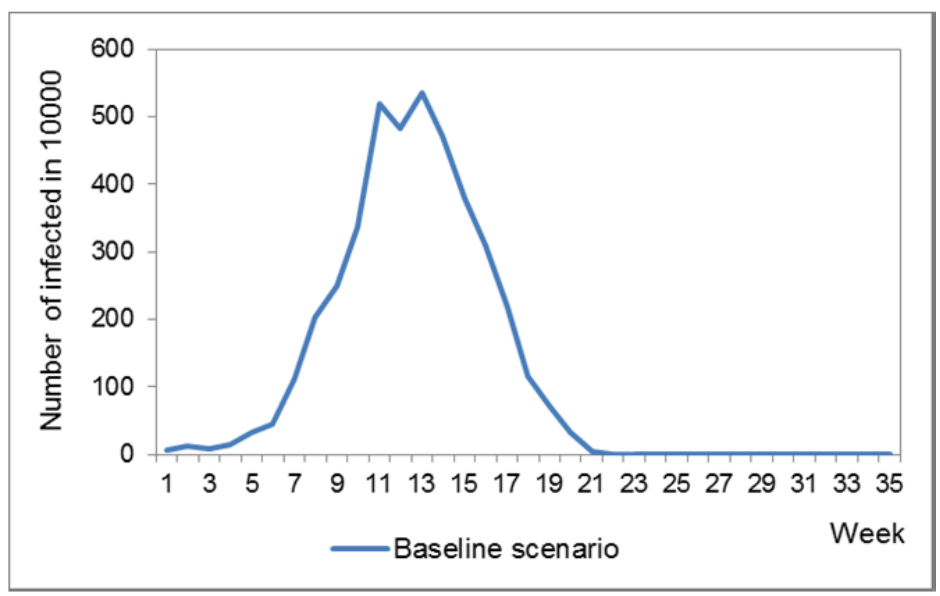

Figure 3: Average weekly infection for baseline scenario

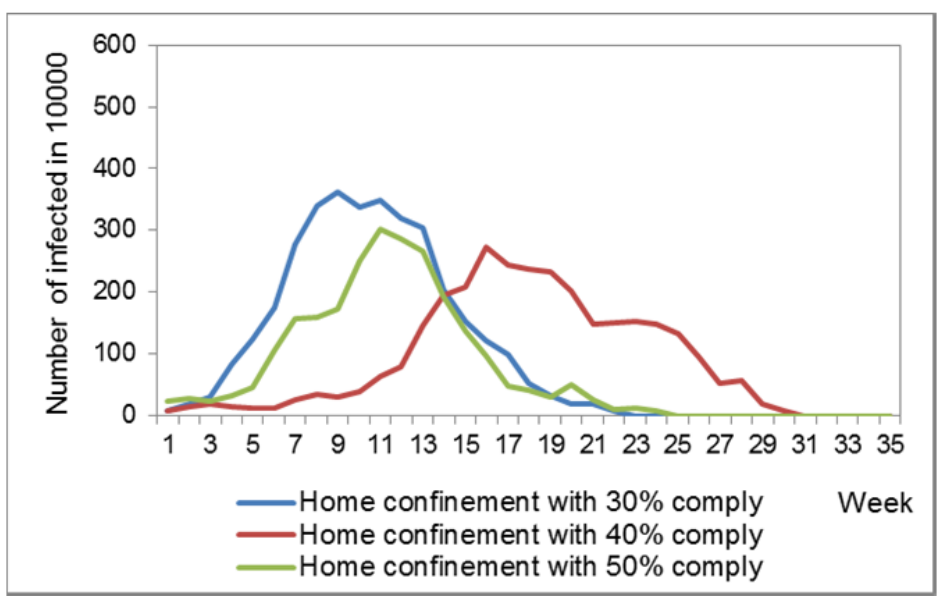

Figure 4: Average weekly infection for home confinement scenario

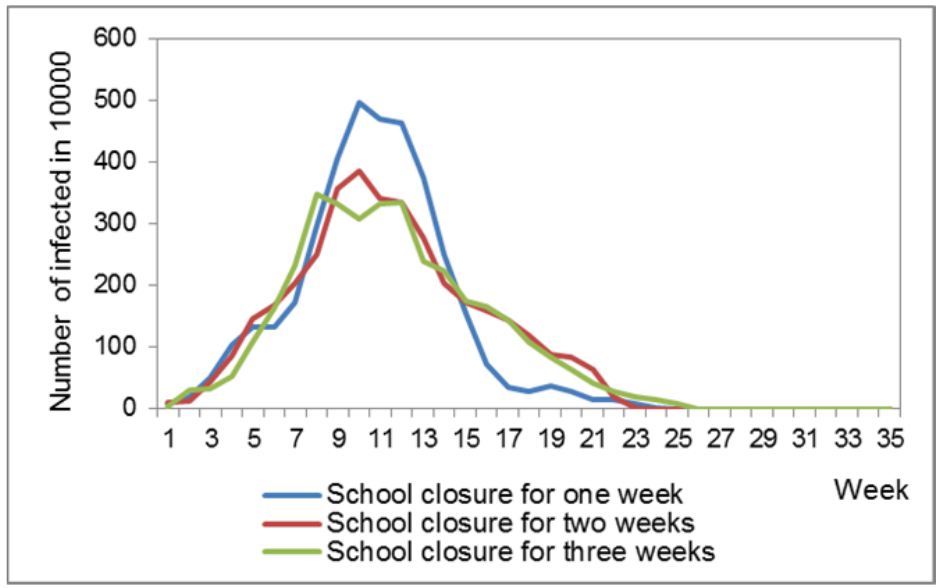

Figure5: Average weekly infection for school closure scenario 
Paleshi, Evans, Heragu, and Moghaddam

Table 2: Attack rate, and effectiveness for each of the scenarios

\begin{tabular}{|c|c|c|c|c|}
\hline Scenario & \multicolumn{2}{|l|}{ Version of scenario } & $\operatorname{AR}(95 \% \mathrm{CI})$ & Effectiveness $(95 \% \mathrm{CI})$ \\
\hline \multirow[t]{2}{*}{ Baseline } & \multirow[t]{2}{*}{-} & - & $41.6(40.7,42.4)$ & - \\
\hline & & 30 & $34.4(33.6,35.2)$ & $17.3(15.4,19.2)$ \\
\hline \multirow[t]{3}{*}{ Home confinement } & \multirow[t]{3}{*}{ Rate of compliance } & 40 & $30.5(29.5,31.5)$ & $26.6(24.1,29.1)$ \\
\hline & & 50 & $25.1(23.9,26.2)$ & $39.6(37.0,42.3)$ \\
\hline & & 1 & $37.5(36.2,38.9)$ & $9.8(6.4,12.9)$ \\
\hline \multirow[t]{2}{*}{ School closure } & \multirow[t]{2}{*}{ Weeks of closure } & 2 & $36.6(35.5,37.6)$ & $12.0(9.4,14.5)$ \\
\hline & & 3 & $35.8(34.7,37.0)$ & $13.9(11.0,16.6)$ \\
\hline
\end{tabular}

\section{SENSITIVITY ANALYSIS}

An infectious person might show disease symptoms (i.e., be symptomatic) or might not show any symptoms (i.e., be asymptomatic). The probability of being symptomatic (PBS) for an infectious person is considered to be equal to $67 \%$ in the literature (Haber et al. 2007; Elveback et al. 1976). The value of PBS can affect the effectiveness of the interventions. A sensitivity analysis is conducted on PBS when a person gets the disease. Here, the attack rate of the pandemic is measured for the baseline scenario, home confinement strategy with $50 \%$ compliance, and three week school closure strategy when PBS is equal to $50 \%, 67 \%$, and $80 \%$. Table 3 shows the AR of the pandemic when each of these intervention strategies is applied.

Table 3: Attack rate and effectiveness for each of the scenarios when PBS is $50 \%, 67 \%$, or $80 \%$

\begin{tabular}{|c|c|c|c|c|c|c|}
\hline & \multicolumn{6}{|c|}{ Probability of being symptomatic } \\
\hline & & $50 \%$ & & $67 \%$ & & $80 \%$ \\
\hline & AR & $\begin{array}{c}\text { Effectiveness } \\
(\%)\end{array}$ & AR & $\begin{array}{c}\text { Effectiveness } \\
(\%)\end{array}$ & $\mathrm{AR}$ & $\begin{array}{c}\text { Effectiveness } \\
(\%)\end{array}$ \\
\hline Baseline & 37.6 & - & 41.6 & - & 45.1 & - \\
\hline $\begin{array}{c}\text { Home confinement } \\
\text { with } 50 \% \text { rate of compli- } \\
\text { ance }\end{array}$ & 21.2 & 43.6 & 25.1 & 39.6 & 30.0 & 33.4 \\
\hline $\begin{array}{c}\text { School closure for } \\
\text { three weeks }\end{array}$ & 31.6 & 15.9 & 35.8 & 13.9 & 38.9 & 13.7 \\
\hline
\end{tabular}

As shown in Table 3, when the probability of being symptomatic is decreased (increased) to $50 \%$ $(80 \%)$ the attack rate for baseline scenario decreases (increases) to $37.6(45.1)$ percent. Further, decreasing (increasing) PBS, decreases (increases) the attack rate when intervention strategies are implemented. Home confinement with 50\% compliance rates works better than school closure for three weeks for all three values of PBS. Moreover, it is shown that the effectiveness levels increase (decrease) when PBS is decreased (increased) for both intervention strategies.

\section{CONCLUSION AND FUTURE RESEARCH}

An agent-based simulation model is developed to investigate the effectiveness of home confinement and school closure intervention strategies on the progression of pandemic influenza. Results of the simulations show that both intervention strategies can be helpful in the mitigation of the pandemic to some extent. A home confinement strategy with $50 \%$ compliance rate decreases the attack rate of the virus by $39.6 \%$ and is the most effective strategy among the ones presented. Further, a sensitivity analysis is conducted to measure the effects of changing the probability of being symptomatic on the effectiveness of the interventions. The results of the simulation show that decreasing (increasing) PBS results in an increase (decrease) in effectiveness of the intervention strategies and a decrease (increase) in the attack rate of the 
pandemic. Future work will include broader versions of home confinement, school closure, and other intervention strategies such as vaccination.

\section{ACKNOWLEDGMENTS}

Funding for this research was provided by the Kentucky Critical Infrastructure Protection Program, managed by The National Institute for Hometown Security for the US Department of Homeland Security.

\section{REFERENCES}

Aleman, D. M., T. G. Wibisono, and B. Schwartz. 2009. "Accounting for Individual Behaviors in a Pandemic Disease Spread Model." In Proceedings of the 2009 Winter Simulation Conference, edited by M. D. Rossetti, R. R. Hill, B. Johansson, A. Dunkin, and R. G. Ingalls, 1977-1985. Piscataway, New Jersey: Institute of Electrical and Electronics Engineers, Inc.

Araz, M. A., J. W. Fowler, T. W. Lant, and M. Jehn. 2009. "A Pandemic Influenza Simulation Model for Preparedness Planning." In Proceedings of the 2009 Winter Simulation conference, edited by M. D. Rossetti, R. R. Hill, B. Johansson, A. Dunkin, and R. G. Ingalls, 1986-1995. Piscataway, New Jersey: Institute of Electrical and Electronics Engineers, Inc.

Dushoff, J., J. B. Plotkin, S. A. Levin, and D. J. D. Earn. 2004. "Dynamical Resonance can Account for Seasonality of Influenza Epidemics." In Proceedings of the National Academy of Sciences of the United States of America 101(48):16915-16916.

Elveback, L. R., J. P. Fox, E. Ackerman, A. Langworthy, M. Boyd, and L. Gatewood. 1976. "An Influenza Simulation Model for Immunization Studies.” American Journal of Epidemiology 103(2):152-165.

Ferguson, N. M., D. A. T. Cummings, C. Fraser, J. C. Cajka, and P. C. Cooley. 2006. "Strategies for Mitigating an Influenza Pandemic." Nature 442(7101):448-452.

Gatherer, D. 2009. "The 2009 H1N1 Influenza Outbreak in its Historical Context." Journal of Clinical Virology 45(3):174-178.

Haber, M. J., D. K. Shay, X. M. Davis, R. Patel, X. Jin, E. Weintraub, E. Orenstein, and W. W. Thompson. 2007. "Effectiveness of Interventions to Reduce Contact Rates during a Simulated Influenza Pandemic." Emerging Infectious Diseases 13(4):581-589.

Hilleman, M. R. 2002. "Realities and Enigmas of Human Viral Influenza: Pathogenesis, Epidemiology and Control." Vaccine 20(25-26):3068-3087.

Longini, I. M., M. E. Halloran, A. Nizam, and Y. Yang. 2004. "Containing Pandemic Influenza with Antiviral Agents." American Journal of Epidemiology 159(7):623-633.

Shi, P., P. Keshinocak, J. L. Swann, and B. Y. Lee. 2010. "Modeling Seasonality and Viral Mutation to Predict the Course of an Influenza Pandemic." Epidemiology and Infection 138(10):472-1481.

Towers, S., and Z. Feng. 2009. "Pandemic H1N1 Influenza: Predicting the Course of a Pandemic and Assessing the Efficacy of the Planned Vaccination Program in the United States." Eurosurveillance 14(41):1-3.

Wu, J. T., S. Riley, C. Fraser, and G. M. Leung. 2006. "Reducing the Impact of the Next Influenza Pandemic Using Household-based Public Health Interventions" PLoS Medicine 3(9):1532-1540.

\section{AUTHOR BIOGRAPHIES}

ARSALAN PALESHI is a Ph.D. Student in the Department of Industrial Engineering at the University of Louisville. He has a B.S. degree in Industrial Engineering and M.S. degree in Social-Economic Systems Engineering from Sharif University of Technology, Iran. He worked as an Industrial Engineer for Ian Khodro Automobile Company. He is currently working on development of real-time decision support system for healthcare and public health. His research interests include, simulation based optimization, 
multi-objective modeling, and healthcare systems. He is vice-president of the INFORMS student chapter at the University of Louisville. He can be reached by e-mail at a0pale01@1ouisville.edu.

GERALD W. EVANS is a Professor in the Department of Industrial Engineering at the University of Louisville. He has a B.S. degree in Mathematics, and M.S. and Ph.D. degrees in Industrial Engineering, all from Purdue University. Before entering academia, he worked as an Industrial Engineer for Rock Island Arsenal, and as a Senior Research Engineer for General Motors Research Laboratories. Besides simulation modeling and analysis, his research interests include multi-objective optimization, decision analysis, and discrete optimization. He is a past Proceedings Editor and Program Chair for the Winter Simulation Conference. He can be reached by e-mail at gwevan01@1ouisville.edu.

SUNDERESH S. HERAGU is Professor and the Mary Lee and George F. Duthie Chair in Engineering Logistics in the Industrial Engineering Department at the University of Louisville. He is also Director of the Logistics and Distribution Institute (LoDI). Previously he was Professor of Decision Sciences and Engineering Systems at Rensselaer Polytechnic Institute. His research interests are in real-time decision support for emergency preparedness for the healthcare and public health and emergency service sectors, supply chain management, design of next generation factory layouts, intelligent agent modeling of automated warehouse systems, application of RFID technology to improve intra-plant and inter-plant logistics, and integration of design and planning activities in advanced logistical systems. He is Principal Investigator for several projects funded by the Department of Homeland security, Defense Logistics Agency, and the National Science Foundation and private industry in many of the above areas. He is an IIE Fellow and received the David F. Baker Distinguished Research Award from the IIE. He serves on the editorial board of 4 industrial engineering journals. He is Senior Vice-President for Publications and a member of the Board of Trustees for IIE, a senior member of IEEE, INFORMS, POMS, and SME. His email address is s.heragu@louisville.edu.

KAMRAN S. MOGHADDAM is an Assistant Professor of Systems Engineering at Southern Polytechnic State University. He received his B.S. in Applied Mathematics from the University of Tehran, M.S. in Industrial Engineering from Tehran Polytechnic and his Ph.D. in Industrial Engineering from the University of Louisville. Over the past 10 years, Dr. Moghaddam has conducted work and research in the field of industrial and systems engineering in a variety of environments. He has been involved in development of simulation and optimization of multi-reservoir system operation, mathematical and computer models for transportation systems, optimal maintenance and replacement scheduling in multi-component systems, and recently real-time decision support system for healthcare and public health protection sponsored by Department of Homeland Security. Dr. Moghaddam's research interests are systems reliability optimization, energy systems optimization, and operations and supply chain management. He can be reached by email atkmoghadd@spsu.edu. 